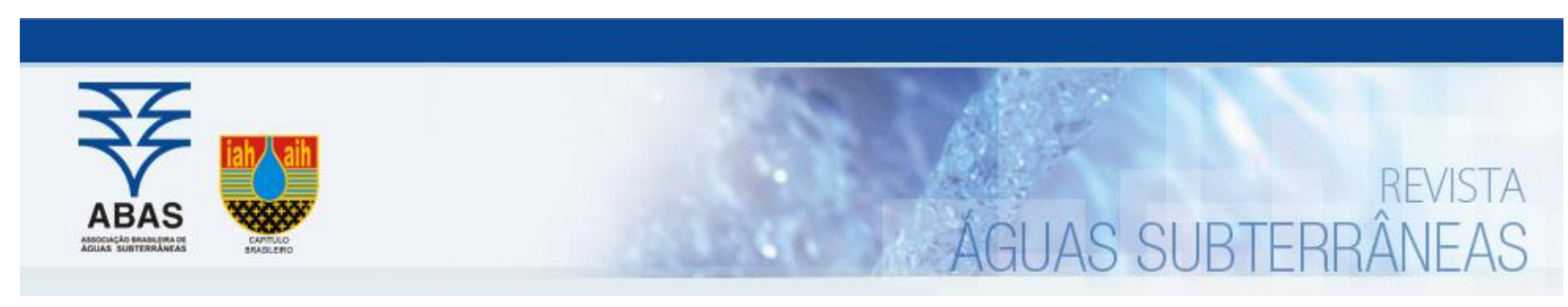

Artigos

\title{
Identificação de adulterações no carvão ativado de osso utilizado na desfluoretação de águas subterrâneas por NIRS-PLS-DA
}

\author{
Identification of adulterations in activated bone charcoal used for \\ groundwater defluoridation by NIRS-PLS-DA
}

\author{
Cristiane Pappis ${ }^{1}$; Marcia Librelotto1; Luiza Baumann ${ }^{1}$ Rosane Bertuzzo1; Ronaldo Bastos dos Santos ${ }^{1}$; Roberta Oliveira Santos ${ }^{1}$; \\ Eduardo Alexis Lobo1; Adilson Ben da Costa ${ }^{1}$
}

1 Universidade de Santa Cruz do Sul (UNISC), Santa Cruz do Sul, RS

\. cristianepappis@outlook.com, marcialibrelotto@mx2.unisc.br, luiza.baumann@gmail.com, rosanebertuzzo1@hotmail.com,

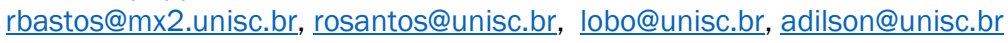

\section{Palavras-chave: \\ Desfluoretação. \\ Carvão ativado de osso bovino. \\ Água para consumo humano.}

Saúde pública.
Keywords:

Defluoridation.

Activated carbon from bovine bone.

Water for human consumption.

Public Health.

Revisão por pares.

Recebido em: 19/08/2020.

Aprovado em: 13/10/2020.

\section{Resumo}

A desfluoretação de águas com excesso de íons fluoreto é fundamental para a saúde pública, visto que de acordo com a Portaria de Consolidação número 05 de 2017 do Ministério da Saúde, a ingestão de água com concentrações de flúor acima de $1,5 \mathrm{mg} \mathrm{L}^{-1}$, pode causar fluorose dentária em crianças. Dentre as técnicas empregadas para a desfluoretação, o método de adsorção usando carvão ativado de osso é um dos mais utilizados. Assim, esta pesquisa teve como objetivo determinar a eficácia do carvão ativado de osso bovino, bem como do carvão vegetal e mineral para a desfluoretação de água, utilizando ferramentas quimiométricas para identificar possíveis adulterações no carvão ativado de osso. Assim, um planejamento fatorial foi realizado para misturar três componentes e testes de adsorção. A espectroscopia no infravermelho próximo (NIRS, do inglês Near Infrared Spectroscopy) foi usada para construir um modelo de análise discriminante por mínimos quadrados parciais (PLS-DA, do inglês Discriminant Analysis by Partial Least Squares). Os resultados mostraram que o carvão ativado de osso é o material mais eficiente para desfluoretação, com capacidade de adsorção de 1,21 $\pm 0,06 \mathrm{mg} \mathrm{g}^{-1}(n=3)$, significativamente superior à capacidade de adsorção do carvão vegetal e mineral $(p<0,05)$. Adulterações no carvão ativado de osso bovino foram identificadas por meio da análise discriminante, indicando que o NIRS-PLS-DA é uma alternativa eficiente para a caracterização e identificação desses adsorventes para sistemas de tratamento de água.

Abstract

The water defluoridation with excess of fluoride ions is essential for public health, considering in accordance to Consolidation Ordinance 05 of 2017 of the Ministry of Health, the water ingestion with fluoride concentrations above $1.5 \mathrm{mg}$ $\mathrm{L}-1$, can cause dental fluorosis in children. Among the techniques used for defluoridation, the adsorption method using activated bone carbon is one of the most used. Thus, this research aimed at determining the effectiveness of activated charcoal from bovine bone, as well as vegetal and mineral charcoal for water defluoridation, using chemometric tools to identify possible adulterations in the activated bone charcoal. Thus, a factorial design was performed to mix three components and adsorption tests. Near-infrared spectroscopy (NIRS) was used to build a model of discriminant analysis by partial least squares (PLS-DA). The results showed that the activated bone charcoal is the most efficient material for defluoridation, with an adsorption capacity of $1.21 \pm 0.06 \mathrm{mg} \mathrm{g}^{-1}(n=3)$, significantly higher than the adsorption capacity of vegetal and mineral charcoal $(p<0.05)$. Adulterations in the activated charcoal from bovine bone were identified using the discriminant analysis, indicating that NIRS-PLS-DA is an efficient alternative for the characterization and identification of these adsorbents for water treatment systems.

DOI: http:/dx.doi.org/10.14295/ras.v34i3.29944

\section{INTRODUÇÃO}

A manutenção da qualidade da água dentro dos parâmetros de potabilidade é uma estratégia de saúde pública fundamental para a manutenção da qualidade de vida de uma população, além de ser um direito fundamental. Esta estratégia é particularmente importante para pequenas comunidades, que por estarem afastadas dos grandes centros urbanos apresentam uma estrutura de assistência à saúde mais precária.

Dente os parâmetros de qualidade monitorados na água de abastecimento público, o flúor tem chamado a atenção dos órgãos de vigilância pela frequência em que são identificados valores em desacordo com os padrões de potabilidade, especialmente em comunidades abastecidas por água de origem 
subterrânea. Isso, associado com a dificuldade técnica de tratamento da água com níveis elevados de íons fluoreto se constituiu hoje num importante problema de saúde pública.

Cabe destacar, que o flúor é um nutriente importante e, quando em níveis adequados atua na prevenção da cárie dental. Porém, quando consumido em grandes quantidades e por um longo período, pode causar uma grave doença que ataca o esmalte dos dentes, a fluorose dental (COSTA, 2010). A Portaria de Consolidação n ${ }^{\circ} 05$ de 2017, do Ministério da Saúde, que trata dos procedimentos de controle e de vigilância da qualidade da água para o consumo humano e sua potabilidade, estabelece que o limite máximo permitido de íons fluoretos em água de abastecimento público deve ser igual a 1,5 mg L-1 (BRASIL, 2017).

Além disso, a ingestão de água com níveis mais elevados de íons fluoretos também está associada a doenças como a osteoporose, artrite, câncer, infertilidade, distúrbios da tireoide e Alzheimer (TIWARI; SINGH; MAHATO, 2017, VINATI; MAHANTY; BEHERA, 2015, WAMBU; MUTHAKIA, 2011).

Nesse contexto, sempre que são detectados níveis elevados de íons fluoretos em águas de abastecimento é fundamental a implementação de sistemas de desfluoretação, para ajustar sua concentração à níveis seguros para o consumo humano.

Várias metodologias já foram propostas para a remoção de flúor, seja por membranas seletivas, troca iônica, reações de precipitação e processos de adsorção (GAO et al., 2009). Entre estas, os processos de adsorção são considerados os mais populares e amplamente utilizadas, devido ao seu baixo custo, eficiência e diversidade de materiais adsorventes disponíveis. Porém, a viabilidade da técnica está relacionada com as características do adsorvente, como seletividade, potencial de regeneração e estabilidade química (YADAV et al., 2018).

Entre os materiais adsorventes pode-se destacar o carvão ativado, que é utilizado em estações de tratamento de água (ETA's), principalmente na remoção de cor e odor, geralmente associada a proliferação de algas nos mananciais de abastecimento. Esses materiais são carbonosos, porosos e se apresentam na forma microcristalina que sofreu um processo de ativação, aumentando a sua porosidade. Quase todos os materiais que possuem um alto teor de carbono podem ser ativados, tanto de origem vegetal (como madeiras, caroços de pêssego, cascas de coco e casca de arroz), mineral (como antracito, betuminoso e linhito), e carvão ativado de osso bovino (SILVA et al., 2012).

Os precursores de origem vegetal ou mineral devem apresentar um teor de carbono $>40 \%$, para assim dar origem a um bom material adsorvente (RASHIDI et al., 2012). Já, o carvão ativado de osso é composto de até $80 \%$ de fosfato tricálcico ou hidroxiapatita $\left(\mathrm{Ca}_{10}\left(\mathrm{PO}_{4}\right)_{6}(\mathrm{OH})_{2}\right), 10 \%$ de carbono e $10 \%$ de $\mathrm{CaCO}_{3}$. Essas características conferem ao carvão ativado de osso uma alta capacidade de remoção de íons fluoreto da água, uma vez que sua rede cristalina atua como um trocador aniônico seletivo para estes íons (RIBEIRO, 2012). Sua estrutura interna possui inúmeros poros, ou sítios vazios, onde os íons fluoreto se ligam e ficam adsorvidos (BONECHAR - Carvão Ativado de Brasil Ltda., 2018).

Nos últimos anos, a desfluoretação de águas subterrâneas utilizando o carvão ativado de osso tem se destacado como uma tecnologia eficiente no tratamento de água subterrânea, seja como meio adsorvente (COSTA et al., 2013; HELFER; COSTA, 2017; PAPPIS et al., 2017; BAUMANN et al. 2018), ou como catalisador em mecanismos de precipitação por contato (COSTA et al., 2013; HELFER; COSTA, 2017; BAUMANN et al., 2018; COSTA, 2018; FUNASA, 2019).

De fato, a composição química do carvão ativado de osso bovino é o que difere dos demais carvões ativados (RIBEIRO, 2012). Desta forma, a utilização de espectroscopia molecular, associada a modelos supervisionados de reconhecimento de padrões, pode contribuir para o controle de qualidade desses materiais, permitindo sua classificação e identificação de misturas e adulterações que podem vir a prejudicar a sua eficiência em sistemas de desfluoretação.

Assim, o objetivo deste estudo foi determinar a efetividade do carvão ativado de osso, carvão ativado mineral e carvão ativado vegetal na desfluoretação de águas subterrâneas, e propor um método de identificação de adulterações do carvão ativado de osso por carvão de origem vegetal e mineral, que se caracterize pela rapidez, baixo custo, não utilizar reagentes químicos e não ser destrutivo, usando a espectroscopia no infravermelho próximo (NIRS, do inglês near infrared spectroscopy) e análise discriminante por mínimos quadrados parciais (PLS-DA, do inglês discriminant analysis by partial least squares).

\section{MATERIAIS E MÉTODOS}

Para o desenvolvimento deste estudo, foram utilizadas amostras de carvão ativado de osso bovino, adquirido junto à empresa Bonechar - Carvão Ativado do Brasil Ltda, e amostras de carvão vegetal e carvão mineral adquiridas junto à empresa Brascarbo Agroindustrial Ltda.

O carvão ativado de osso, teve como matéria prima, ossos bovinos triturados, o qual foi ativado fisicamente à elevadas temperaturas, adquirindo uma alta área superficial interna de 120 $\mathrm{m}^{2} \mathrm{~g}^{-1}$, formada por milhares de poros de tamanhos que variam entre 7,5 a $60.000 \mathrm{~nm}$. Já os carvões ativados de origem vegetal e mineral tiveram como materiais precursores, cascas de coco e carvão mineral betuminoso, respectivamente, sendo ativados fisicamente com vapor a altas temperaturas. As demais especificações destes materiais estão apresentadas na Tabela 1.

Para uniformização do tamanho das partículas dos materiais, todas as amostras de carvão foram secas a $60^{\circ} \mathrm{C}$ por 12 horas, moídas com o auxílio de um almofariz e pistilo e, peneiradas em malha de 20 mesh. 
Tabela 1 - Caracterização dos carvões ativados utilizados neste estudo

\begin{tabular}{|c|c|c|c|}
\hline Características & Carvão ativado de osso & Carvão vegetal & Carvão mineral \\
\hline Matéria prima & Ossos bovinos triturados & Cascas de coco & Carvão mineral betuminoso \\
\hline Ativação & física & física & física \\
\hline Tamanho de poro & 7,5 a $60.000 \mathrm{~nm}$ & - & - \\
\hline pH & $8,5-9,5$ & 8,0 & 8,0 \\
\hline Densidade aparente & $0,60-0,70 \mathrm{~g} \mathrm{~cm}^{-3}$ & $\begin{array}{l}0,45-0,55 \mathrm{~g} \\
\mathrm{~cm}^{-3}\end{array}$ & $0,45-0,55 \mathrm{~g} \mathrm{~cm}^{-3}$ \\
\hline Umidade & $<5 \%$ & $\leq 10 \%$ & $\leq 3 \%$ \\
\hline Dureza & $>80$ & $>90$ & $>90$ \\
\hline
\end{tabular}

\subsection{Determinação da capacidade de adsorção}

Para a determinação da capacidade de adsorção de íons fluoretos foram seguidas etapas de procedimentos, conforme apresentado no fluxograma da Figura 1 , com 0,5 g de cada amostra de carvão puro, e adulterados. Todos os ensaios foram feitos em triplicata. As amostras de carvão ativado de osso foram adulteradas pela adição de quantidades iguais de carvão vegetal e mineral, formando misturas binárias e ternárias $(50 \% \mathrm{~m} / \mathrm{m}$ e $33,3 \% \mathrm{~m} / \mathrm{m})$. As amostras de carvão foram transferidas para erlenmeyers de $250 \mathrm{~mL}$, contendo $200 \mathrm{~mL}$ de solução de referência de fluoreto de sódio PA (Synth®, Diadema, SP), de 5,0 mg L-1. Imediatamente após o preparo, os erlenmeyers foram dispostos em um agitador orbital e agitados por 360 minutos a $240 \mathrm{rpm}$, conforme Alessio et al. (2014).
Transcorrido o tempo de agitação a concentração final de íons fluoretos em cada ensaio foi determinada por potenciometria direta, utilizando um sensor de íon seletivo para flúor (APHA, 2005).

A capacidade de adsorção (CA) dos materiais adsorventes foi determinada conforme a Eq. 1.

$C A=\frac{\left\{\left[C_{i}-C_{f}\right]\right\} \cdot V}{m}$

Onde: $\mathrm{CA}$ - capacidade de adsorção (mg g-1); $\mathrm{C}_{\mathbf{i}}$ - concentração inicial $\left(m g L^{-1}\right) ; C_{f}$ - concentração final $\left(m g L^{-1}\right) ; V$ - volume da solução (L); m - massa de carvão (g).

Figura 1 - Fluxograma da sequência dos procedimentos para a determinação da capacidade de adsorção dos materiais

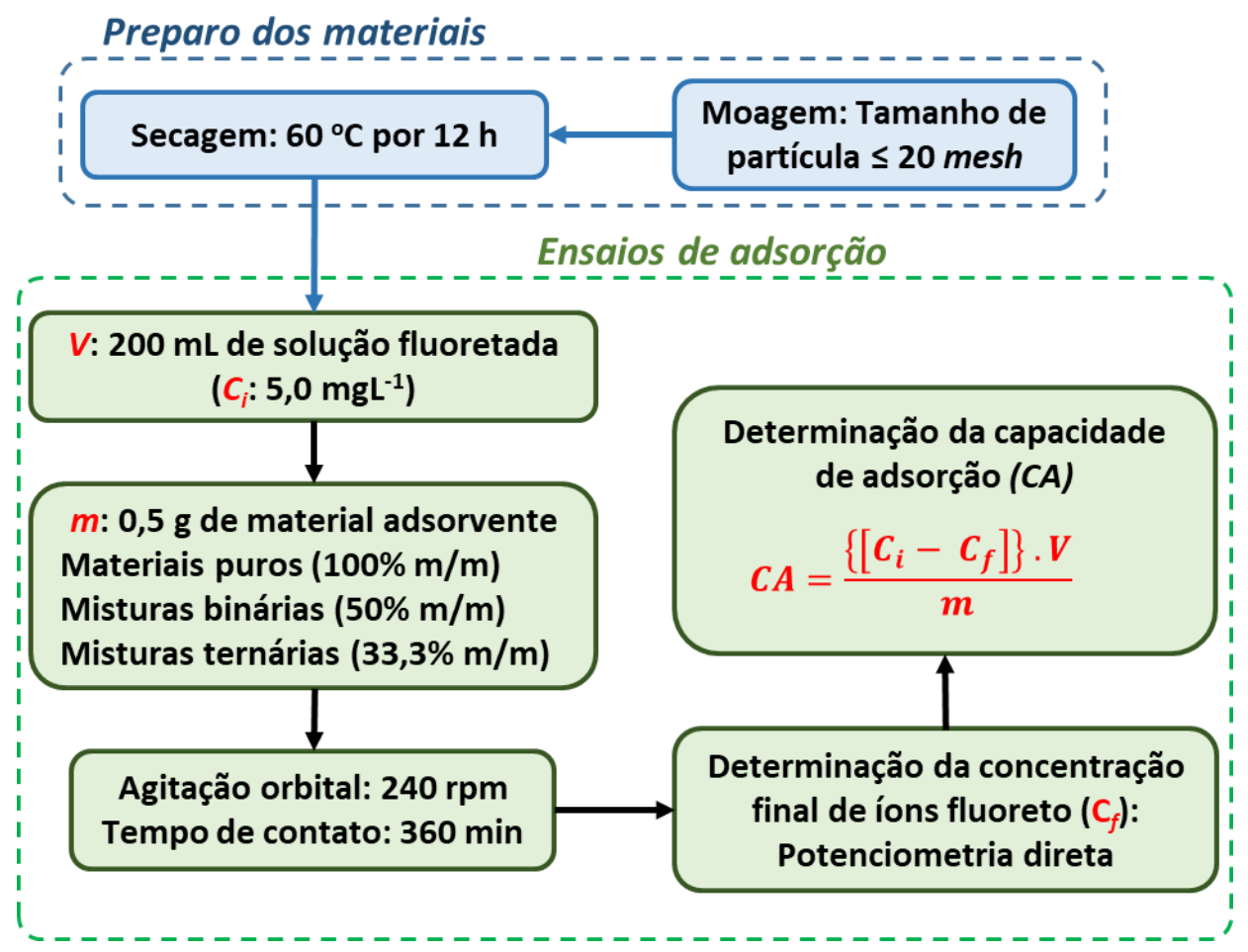

\subsection{Planejamento experimental}

Para identificar as influências das variáveis experimentais e seus efeitos na capacidade de adsorção das amostras, foi ela- borado um planejamento fatorial para misturas de três componentes, com auxílio do Software Chemoface ${ }^{\circledR}$ versão 1.64 . As informações de composição das misturas dos materiais adsorventes encontram-se na Tabela 2. 
Tabela 2 - Composições das misturas de carvão obtidas no planejamento fatorial de três componentes

\begin{tabular}{cccc}
\hline \multicolumn{4}{c}{ Proporção dos materiais das misturas $(\% \mathrm{~m} / \mathbf{m})$} \\
\hline Experimentos & Carvão ativado de osso & Carvão vegetal & Carvão mineral \\
\hline 1 & 100 & 0 & 0 \\
3 & 0 & 100 & 0 \\
4 & 0 & 0 & 100 \\
5 & 50 & 50 & 0 \\
6 & 50 & 0 & 50 \\
7 & 0 & 50 & 50 \\
\end{tabular}

\subsection{Aquisição dos espectros de NIRS e tratamento dos da-} dos

Para a aquisição dos espectros foram usadas amostras dos carvões puros (A - carvão ativado de osso; V - carvão vegetal; e M - carvão mineral; misturas binárias (A-V; A-M; e V-M, 50\% $\mathrm{m} / \mathrm{m})$; e uma mistura ternária (A-M-V, $33 \% \mathrm{~m} / \mathrm{m})$, e duas amostras A-V e A-M, com proporções de adulteração de 10 , 20, 30 e 40\%. 10 g de carvão foram utilizadas para cada análise, as quais foram transferidas para frascos de poliestireno de $75 \mathrm{~mL}$ e dispostas em uma mesa auto-amostradora do tipo X, Y, Z modelo FZ 100-PRT, Fooze Tecnologia ${ }^{\circledR}$ (Almirante Tamandaré, Brasil). Os espectros foram adquiridos na região do infravermelho próximo (951 - $2450 \mathrm{~nm}$ ), com área de varredura de $4 \mathrm{~cm}^{2}$ sobre a superfície da amostra e resolução de $3,2 \mathrm{~nm}$. Os espectros foram adquiridos em quintuplicata $(n=5)$.

Os espectros foram transferidos para uma planilha eletrônica Microsoft Excel ${ }^{\circledR}$ e tratados utilizando o software SOLO + MIA (Eigenvector Research, Inc), versão 8.6.1 (2018). Para a identificação e caracterização do carvão ativado de osso e suas adulterações foi realizada a análise supervisionada de reconhecimento de padrões, utilizando PLS-DA (FERREIRA, 2015).

\section{RESULTADOS}

\subsection{Capacidade de adsorção dos carvões}

A Tabela 3 apresenta os resultados dos ensaios de adsorção para cada amostra de carvão, utilizando uma solução fluoretada com concentração de 4,98 mg L-1.

Tabela 3 - Resultados dos ensaios realizados com as amostras de carvão ativado de osso, carvão vegetal, carvão mineral e suas misturas

\begin{tabular}{lcccc} 
& \multicolumn{2}{c}{$\begin{array}{c}\text { Concentração final de íons fluoreto } \\
\text { (mg L-1) }\end{array}$} & $\begin{array}{c}\text { Média } \pm \text { desvio } \\
\text { padrão (mg L-1) }\end{array}$ \\
\cline { 2 - 4 } & Ensaio 1 & Ensaio 2 & Ensaio 3 \\
\hline Carvão ativado de osso (A) & 1,74 & 2,12 & 1,88 & $1,93 \pm 0,19$ \\
Carvão vegetal (V) & 4,75 & 4,95 & 4,86 & $4,85 \pm 0,08$ \\
Carvão mineral (M) & 4,98 & 4,96 & 4,93 & $4,96 \pm 0,02$ \\
Carvão ativado de osso + carvão vegetal & 2,97 & 3,01 & 2,90 & $2,96 \pm 0,05$ \\
Carvão ativado de osso + carvão mineral & 3,02 & 2,95 & 3,03 & $3,00 \pm 0,04$ \\
Carvão vegetal + carvão mineral & 4,93 & 4,93 & 4,97 & $4,94 \pm 0,02$ \\
Carvão ativado de osso + carvão vegetal + carvão mineral & 3,61 & 3,57 & 3,54 & $3,57 \pm 0,03$ \\
\hline
\end{tabular}

Concentração inicial de íons fluoreto $=4,98 \mathrm{mg} \mathrm{L}^{-1}$

A partir dos resultados encontrados, foi possível verificar que os ensaios que obtiveram maior redução na concentração de íns fluoretos foram os que continham o carvão ativado de osso bovino, atingindo até $61,2 \%$ de eficiência, para os ensaios com carvão de osso puro (A). Já as demais amostras de carvão obtiveram uma taxa de remoção inferior, assim como suas respectivas misturas.

A partir da determinação da concentração final de íons fluoretos nos respectivos ensaios foi possível determinar a capaci- dade de adsorção do carvão ativado de osso, do carvão vegetal, carvão mineral e suas respectivas misturas, conforme apresentado na Figura 2. Os valores obtidos para a capacidade de adsorção comprovam que o carvão ativado de osso é o material mais apropriado para ser aplicado em projetos de remoção do excesso de flúor, apresentando uma capacidade média de adsorção de 1,21 $\pm 0,06 \mathrm{mg} \mathrm{g}^{-1}(\mathrm{n}=3)$. Além disso, se observa que a adulteração do carvão de osso por carvão de origem vegetal ou mineral reduz significativamente $(p<0,05)$ a capacidade de adsorção de íons fluoreto. 
Figura 2 - Resultados médios $(n=3)$ da capacidade de adsorção $(C A)$ das amostras de carvão ativado de osso (A), carvão vegetal (V) e carvão mineral (M), e suas misturas

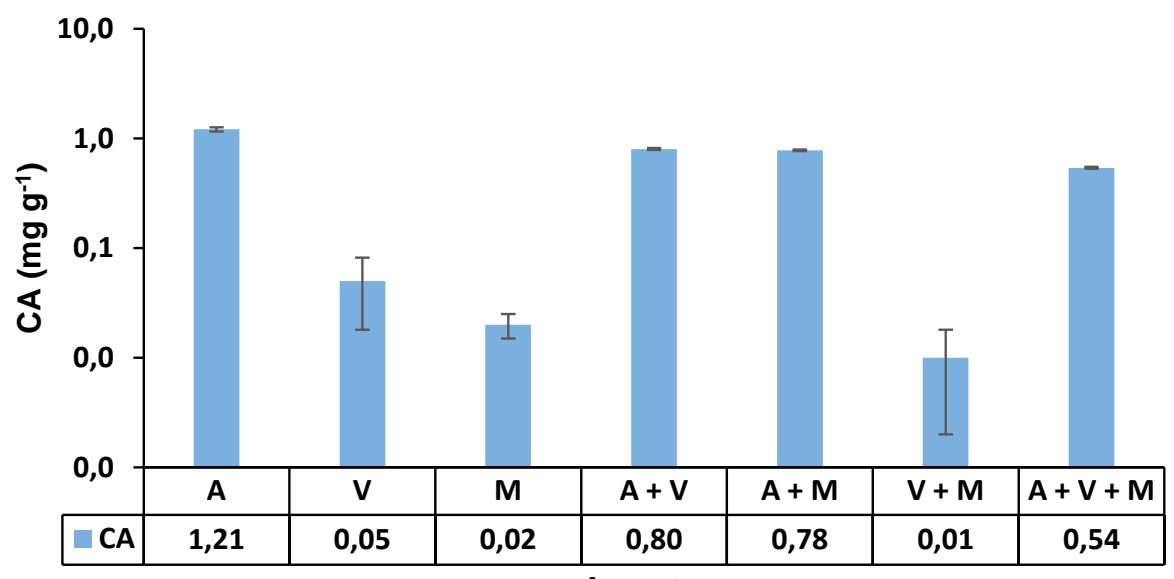

Os resultados obtidos para a capacidade de adsorção utilizando o carvão ativado de osso assemelham-se aos encontrados na literatura. Alessio et al. (2014) obtiveram resultado de capacidade de adsorção de $1,2 \mathrm{mg} \mathrm{g}^{-1}$ ao utilizar carvão ativado de osso com tamanho de 20x60 mesh, e Costa et al. (2013), que usou o carvão ativado de osso bovino de tamanho de 8 a 30 mesh, obtiveram resultados de capacidade de adsorção de 0,79 a $1,6 \mathrm{mg} \mathrm{g}^{-1}$.

\subsection{Planejamento experimental para mistura de três compo- nentes}

Os resultados encontrados no teste de significância para os componentes do modelo (Tabela 4), demonstraram que os resultados de adsorção do carvão ativado de osso $\left(X_{A}\right)$, carvão vegetal $\left(X_{V}\right)$, mistura de carvão ativado de osso e carvão vegetal $\left(X_{A} * X_{V}\right)$ e a mistura de carvão ativado de osso e carvão mineral $\left(X_{A} * X_{M}\right)$ foram considerados significativos $(p<0,05)$ para o modelo quadrático.

$\underline{\text { Tabela } 4 \text { - Teste de significância para os coeficientes do modelo quadrático }}$

\begin{tabular}{|c|c|c|c|c|c|}
\hline & b & Erro & $\mathrm{t}$ & $p$ & Significância \\
\hline$A\left(X_{A}\right)$ & 1,21 & 0,0192 & 62,9969 & 0 & sim \\
\hline $\mathrm{V}\left(\mathrm{X}_{\mathrm{v}}\right)$ & 0,050 & 0,0192 & 2,6032 & 0,0208 & sim \\
\hline $\mathrm{M}\left(\mathrm{X}_{\mathrm{M}}\right)$ & 0,0147 & 0,0192 & 0,7636 & 0,4578 & não \\
\hline$X_{A} * X_{V}$ & 0,6867 & 0,0941 & 7,2975 & $3,92 \times 10^{-6}$ & $\operatorname{sim}$ \\
\hline$X_{A} * X_{M}$ & 0.6867 & 0,0941 & 7,2975 & $3,92 \times 10^{-6}$ & sim \\
\hline$X v * X_{M}$ & $-0,080$ & 0,0941 & $-0,8502$ & 1,5905 & não \\
\hline$X_{A} * X_{V} * X_{M}$ & $-0,6280$ & 0,6620 & $-0,9486$ & 1,6411 & não \\
\hline
\end{tabular}

Onde: $\mathrm{b}$ - valores de resposta para os componentes puros; $\mathrm{t}$ - $\mathrm{t}$ crítico; $\mathrm{p}$ - Número de coeficientes.

Desta forma, os valores de resposta (b) para os componentes foram utilizados para propor um modelo de regressão para o planejamento fatorial de misturas de três componentes através da Eq. 2 (PEREIRA FILHO, 2017), que considerando apenas os efeitos significativos resulta na Eq. 3.

$\hat{Y}=b_{A} X_{A}+b_{V X V}+b_{M} X_{M}+b_{A V X_{A} X_{V}}+b_{A M X_{A} X_{M}}+b_{V M} X_{V} X_{M}+$ $b_{A V M X A X V X M}$

$\hat{Y}=1,21 \cdot 1+0,05 \cdot 1+0,0147 \cdot 1+0,6867 \cdot 0,5 \cdot 0,5+$ $0,6867.0,5.0,5-0,08 \cdot 0,5.0,5-0,628.0,33.0,33$

$\hat{Y}=b_{A} X_{A}+b v X_{V}+b_{A V X A X V}+b_{A M X_{A} X_{M}}$

$\hat{Y}=1,21 \cdot 1+0,05 \cdot 1+0,6867 \cdot 0,5 \cdot 0,5+0,6867 \cdot 0,5 \cdot 0,5$
Através da análise estatística do modelo quadrático gerado (Tabela 5), é possivel verificar que a regressão proposta é significativa, com nível de confiança de 95\%, não havendo evidências de falta de ajuste. Deste modo, o modelo pode ser empregado satisfatoriamente. 
Tabela 5 - Análise de variância para ajuste do modelo quadrático para mistura de três componentes

\begin{tabular}{l|ccccc}
\hline & SQ & GL & MQ & F & p \\
\hline Regressão & 4,0779 & 3 & 1,3593 & 1254,2 & 0 \\
Resíduo & 0,0184 & 17 & 0,0011 & & 0,4739 \\
Falta de ajuste & 0,0029 & 3 & 0,0010 & 0,8824 & \\
Erro puro & 0,0155 & 14 & 0,0011 & & \\
Total & 4,0737 & 20 & & & \\
R $^{2}$ & 1,0010 & & & & \\
Variação explicada R2 & 0,9962 & & & & \\
\hline
\end{tabular}

Onde: $\mathrm{R}^{2}$ - coeficiente de correlação; $\mathrm{SQ}$ - soma quadrática; $\mathrm{GL}$ - graus de liberdade; $\mathrm{MQ}$ - média quadrática; $\mathrm{F}$ - teste f; $\mathrm{p}$ - número de coeficientes.

A aplicação da Eq. 3 permitiu gerar uma superfície de resposta (Figura 3), que ilustra a resposta em função da composição das misturas dos carvões, auxiliando também na definição do ponto ótimo, que corresponde ao ponto no qual se tem a maior capacidade de adsorção da mistura (CA). Assim, é possível identificar que os maiores resultados de capacidade de adsorção são obtidos em misturas mais ricas em carvão ativado de osso.

Figura 3 - Superfície de resposta do modelo quadrático de capacidade de adsorção para os carvões ativados puros e suas respectivas misturas.

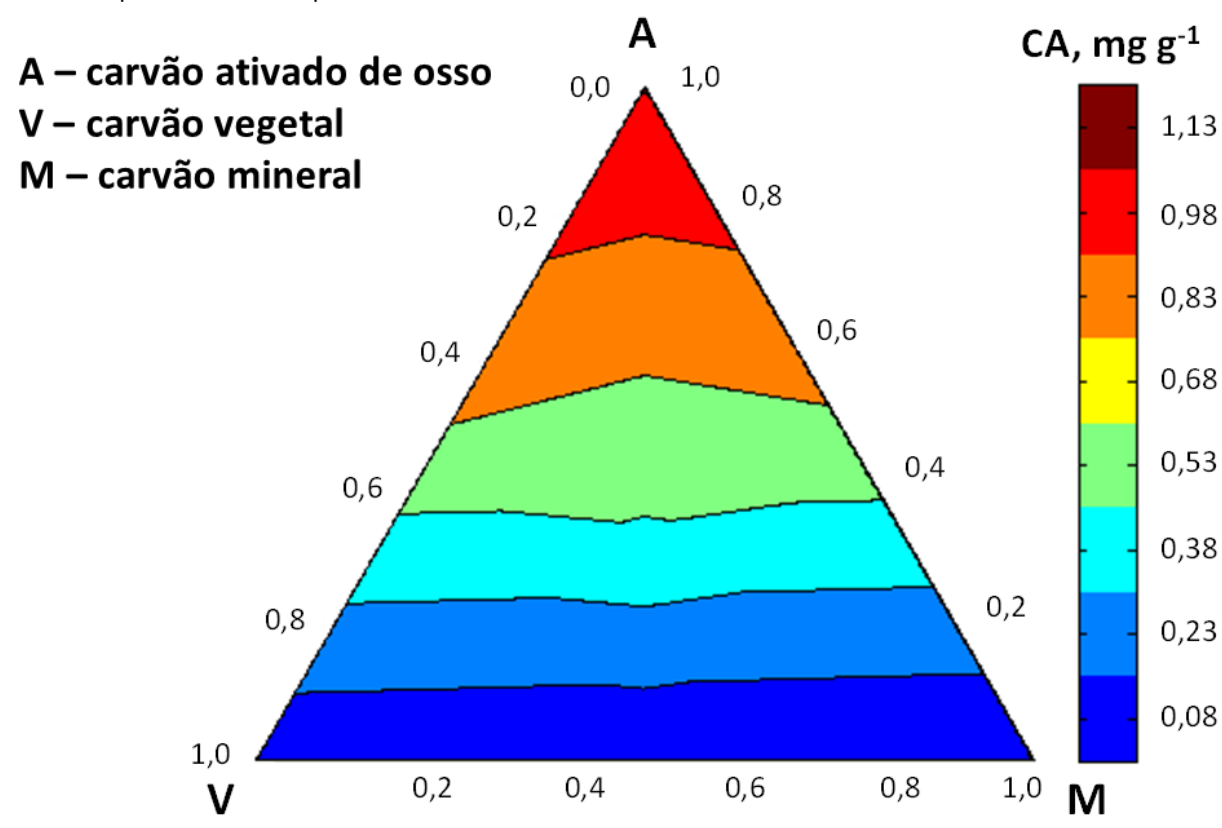

O fato de o carvão vegetal e mineral não possuírem propriedades adsortivas para íons fluoreto está relacionado a sua composição química. Segundo Ribeiro (2012), os carvões vegetal e mineral são ricos em carbono, já o carvão ativado de osso possui apenas $10 \%$ de carbono em sua estrutura e 70 a $75 \%$ de hidroxiapatita $\left(\mathrm{Ca}_{10}\left(\mathrm{PO}_{4}\right)_{6}(\mathrm{OH})_{2}\right)$, além de quantidades de calcita $\left(\mathrm{CaCO}_{3}\right)$ formada devido a presença de $\mathrm{CO}_{2}$ no forno durante a sua produção. Assim quando a hidroxiapatita presente no carvão ativado de osso entra em contato com os íons fluoretos da água, ela atua como um trocador aniônico seletivo para íons fluoreto formando a fluorapatita e mais duas hidroxilas, conforme a reação abaixo:

$\mathrm{Ca}_{10}\left(\mathrm{PO}_{4}\right)_{6}(\mathrm{OH})_{2}+2 \mathrm{~F}^{-} \rightarrow \mathrm{Ca}_{10}\left(\mathrm{PO}_{4}\right)_{6} \mathrm{~F}_{2}+2 \mathrm{OH}^{-}$

\subsection{Análise Discriminante por Mínimos Quadrados Parciais}

O perfil dos espectros de NIRS das amostras puras de carvão ativado de osso, carvão vegetal e carvão mineral estão apresentados na Figura 4. Conforme Andrés \& Bona (2005), os espectros de carvão na região NIRS não apresentam absorções de alta intensidade. E devido a efeitos físicos, como o tamanho de partícula da amostra, índice de refração, forma, distribuição e a efeitos químicos, resultam em sobreposições e muitas vezes em picos amplos. Devido ao espectro NIRS ser o resultado de absorbâncias de tons excessivos e combinações de absorbâncias de vários grupos funcionais (tais como $\mathrm{CH}$, $\mathrm{NH}$ e $\mathrm{OH}$ ) o uso de métodos analíticos de dados multivariados é necessário para revelar informações especificas e uteis (ANDRÉS e BONA, 2005). 
A sequência de pré-tratamentos espectrais que apresentou os melhores resultados foi selecionado como: primeira derivada, padronização normal do sinal (SNV, do inglês standard normal variate) e centragem dos dados na média ( $\mathrm{MC}$, do inglês mean center). 0 modelo foi desenvolvido com 3 variáveis latentes.

Figura 4 - Espectros NIRS sem pré-tratamento $(n=5)$ das amostras puras de carvão ativado de osso, carvão animal e carvão vegetal

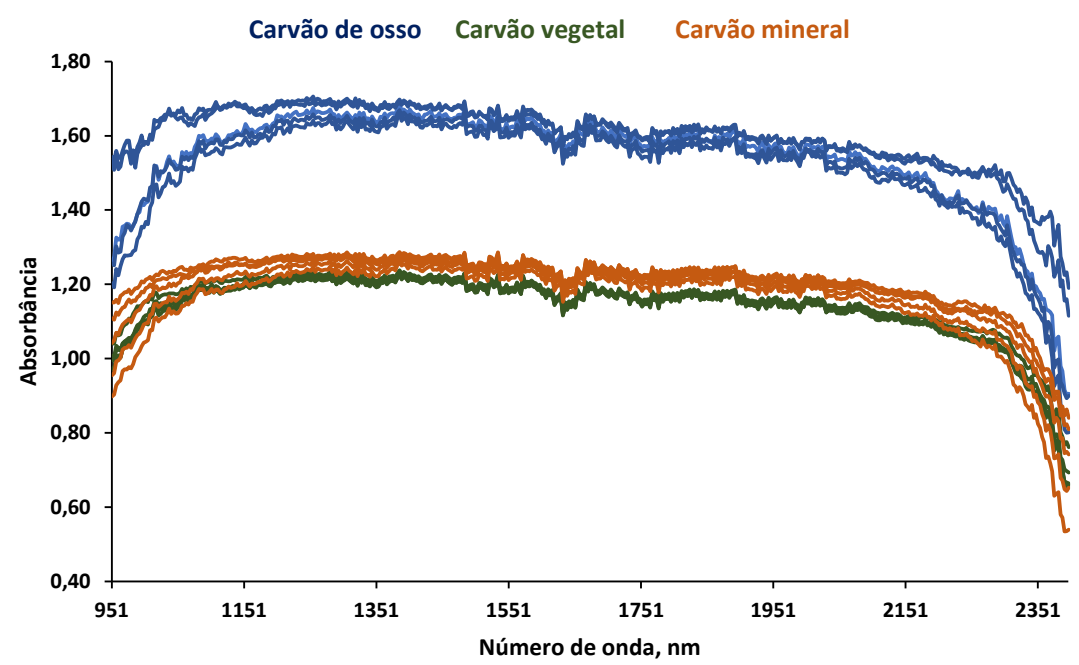

Como pode ser observado no gráfico de scores (Figura 5), o modelo classificou corretamente as amostras de carvão ativado de osso bovino (vermelho), uma vez que estas amostras ficaram acima do limite (threshold), das amostras adulteradas com carvão vegetal e mineral (verde). 0 modelo apresentou resultados de sensitividade e especificidade de calibração de 1,00 e 0,98 , respectivamente. Os resultados de validação cruzada apresentaram resultados de sensitividade e especificidade de 1,00 e 0,94 , respectivamente.

Figura 5 - Scores do modelo de PLS-DA para classificação das amostras de carvão ativado de osso puro e adulterado com carvão de origem vegetal e mineral

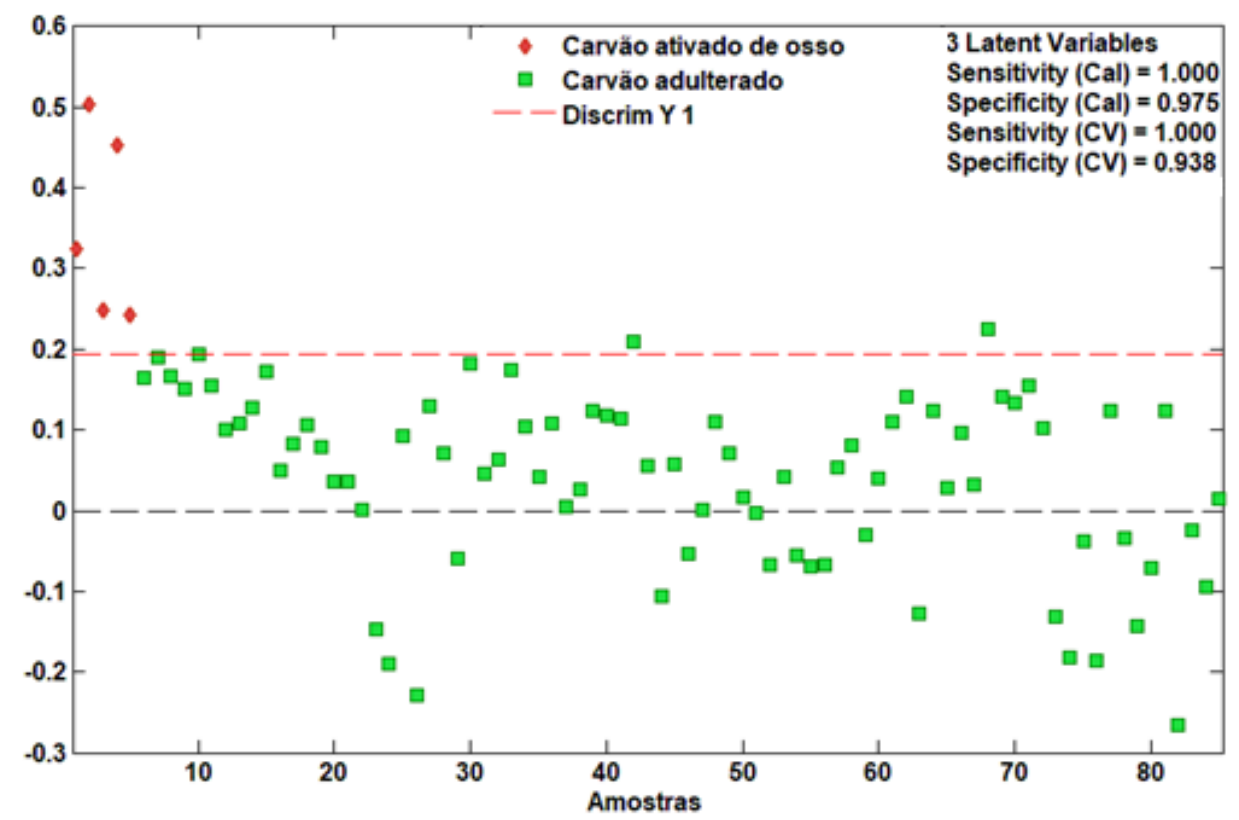

Assim, é possível identificar no modelo que todas as amostras de carvão ativado de osso foram classificadas corretamente, indicando que a metodologia de identificação de adulterações no carvão ativado de osso por NIRS-PLS-DA é uma alternativa promissora para o controle de qualidade destes materiais. Além disto, a metodologia proposta apresenta como características a rapidez, baixo custo, não utilizar reagentes químicos e não ser destrutiva. 


\section{CONCLUSÃO}

Este estudo permitiu demostrar experimentalmente que o carvão ativado de osso, quando comparado com o carvão vegetal e mineral, é o material que possui propriedades mais adequadas para a remoção de íons fluoreto de águas. Todas as amostras de carvão ativado de osso que foram adulteradas com carvão mineral e vegetal, tiveram uma redução na sua capacidade total de adsorção, a qual pode ser comprovada também pelo modelo quadrático de mistura para três componentes.

A utilização da NIRS-PLS-DA permitiu identificar amostras de carvão ativado de osso adulteradas com carvão vegetal e mineral. Com isso, a metodologia analítica proposta demonstrou ser eficiente na identificação de material adsorvente adulterados, podendo ser utilizada nas indústrias e em órgãos de controle e tratamento de águas, com a finalidade de caracterização dos materiais utilizados em sistemas de tratamento de água para abastecimento público.

\section{REFERÊNCIAS}

ALESSIO, K. O. et al. Desfluoretação parcial de águas subterrâneas por adsorção/precipitação por contato em microcolunas de filtração. Jovens Pesquisadores, v. 4, n. 3, p. 53-66, 2014. http://dx.doi.org/10.17058/rip.v4i3.4510

ANDRÉS, J. M.; BONA, M. T. Analysis of coal by diffuse reflectance near-infrared spectroscopy. Analytica chimica acta, v. 535, n. 1-2, p. 123-132, 2005 https://doi.org/10.1016/i.aca.2004.12.007

APHA - American Public Health Association, American Water Works Association AWWA, Water Environment Federation WEF. Standard methods for the examination of water \& wastewater, Washington: APHA, 21 ed., 1000p., 2005.

BAUMANN, L. et al. Aplicação do software Defluoridation no dimensionamento do sistema de desfluoretação de águas subterrâneas utilizando carvão ativado de osso granulado. Águas Subterrâneas, v. 32, n. 1, p. 61-69, 2018. http://dx.doi.org/10.14295/ras.v32i1.29089

BONECHAR, Carvão Ativado do Brasil Ltda. Carvão ativado de osso. Disponivel em: https://www.bonechar.com.br/carvaoativado. Acesso em: 18 ago. 2020.

BRASCARBO, Agroindustrial Ltda. Carvão ativado. Disponível em: http://www.brascarbo.com.br/index.html. Acesso em: 18 ago. 2020 .

BRASIL, Portaria de Consolidação $n^{\circ} 5$, de 28 de setembro de 2017. Ministério da Saúde, MS. Consolidação das normas sobre as ações e os serviços de saúde do Sistema Único de Saúde, 2017 Disponível em: https://portalarquivos2.saude.gov.br/images/pdf/2018/marco/29/PRC-5-Portaria-de-Consolida----o-n---5--de-28-de-setembro-de-2017.pdf. Acesso em: 18 ago. 2020.

CHEMOFACE. Free user-friendly interface for Chemometrics. Disponivel em: http://www.ufla.br/chemoface/. Acesso em: 18 ago. 2020.

COSTA, A. B. (Org.). Água \& Saúde. 1 ed. Santa Cruz do Sul: EDUNISC, 2010. 115 p. ISBN 978-85-7578-269-9.
COSTA, A. B., et al. Desfluoretação de águas subterrâneas utilizando filtros de carvão ativado de osso. Águas Subterrâneas, v. 27, n.3, p. 60-70, 2013. https://doi.org/10.14295/ras.v27i3.27382

COSTA, A. B., et al. Manual para remoção do excesso de flúor em águas naturais. $1 \mathrm{ed}$. Universidade de Santa Cruz do Sul: UNISC, Santa Cruz do Sul, 2018. ISBN: 978-85-98355-15-3.

FERREIRA, M. M. C. Quimiometria: conceitos, métodos e aplicações. Campinas: Editora UNICAMP, 2015. 493 p. ISBN 978 85-268-1063-1.

FUNASA - Fundação Nacional de Saúde. $9^{\circ}$ Caderno de Pesquisa da FUNASA / Ministério da Saúde. 1 ed. Brasília: Ministério da Saúde, 256 p., 2018. ISBN 978-85-7346-055-1.

GAO, S. et al. Size-dependent defluoridation properties of synthetic hydroxyapatite. Journal of Fluorine Chemistry, v. 130, n.6, p. 550-556, 2009 . https://doi.org/10.1016/i.jfluchem.2009.03.007.

Helfer, G. A.; Costa, A. B. Software Defluoridation. Registro no INPI BR 512017000697-0, Programa de Mestrado em Sistemas e Processos Industriais PPGSPI, UNISC, 2017. Disponivel em: http://www.unisc.br/ppgspi. Acesso em: 29 set. 2020.

PAPPIS, C. et al. Monitoramento da qualidade da água tratada por sistema de desfluoretação de águas subterrâneas do campus da Universidade de Santa Cruz do Sul (UNISC), cidade de Santa Cruz do Sul, RS, Brasil. Jovens Pesquisadores, v. 7 , n. 1, p. 18-29, 2017. http://dx.doi.org/10.17058/rip.v7i1.9307.

PEREIRA FILHO, E. R. Planejamento fatorial em química: maximizando a obtenção de resultados. 1 ed. São Carlos: Edufscar, 2017. 88 p. ISBN 978-85-7600-397-7.

RASHIDI, N. A. et al. Activated carbon from the renewable agricultural residues using single step physical activation: A preliminary analysis. APCBEE Procedia, v. 3, p. 84-92, 2012. https://doi.org/10.1016/i.apcbee.2012.06.051.

RIBEIRO, M. $V$ et al. Bonechar: an alternative for removal of diverse organic and inorganic compounds from industrial wastewater. In: Amit Bhatnagar. (Org.). Application of Adsorbents for Water Pollution Control. 1 ed. Brussels: Bentham Science Publishers, 2012. 502-522 p. ISBN 978-1-60805-5388.

SILVA, G. G. et al. Tratamento de água de reservatórios por dupla filtração, oxidação e adsorção em carvão ativado granular. Engenharia Sanitária e Ambiental, v. 17, n. 1, p. 71-80, 2012.

41522012000100011. https://doi.org/10.1590/S1413

SOLO + MIA. Eingenvector Research. Hyacinth, Manson. Disponível em: https://eigenvector.com/software/solo+mia.htm. Acesso em: 18 ago. 2019.

TIWARI, A., SINGH, A. MAHATO, D. M. GIS based evaluation of fluoride contamination and assessment of fluoride exposure dose in groundwater of a district in Uttar Pradesh, India. Human and Ecological Risk Assessment, v. 23, p. 56-66, 2017. https://doi.org/10.1080/10807039.2016.1220824

VINATI, A., MAHANTY, B. BEHERA, S. Clay and Clay Minerals for Fluoride Removal from Water: A State-of-the-Art Review. 
Applied Clay Science, v. 144, p. 340-348, 2015. https://doi.org/10.1016/i.clay.2015.06.013

WAMBU, E. MUTHAKIA, G. High Fluoride Water in the Gilgil Area of Nakuru County, Kenya. Research report Fluoride, v. 44, n. 1, p. 37-41, 2011.
YADAV, K. K. et al. A review of emerging adsorbents and current demand for defluoridation of water: Bright future in water sustainability. Environment International, v. 111, p. 80-108, 2018. https://doi.org/10.1016/j.envint.2017.11.014. 